\title{
Slender Ca II H Fibrils Mapping Magnetic Fields in the Low Solar Chromosphere
}

\author{
S. Jafarzadeh ${ }^{1}$, R. J. Rutten ${ }^{1}$, S. K. Solanki ${ }^{2,3}$, T. Wiegelmann ${ }^{2}$, T. L. Riethmüller ${ }^{2}$, M. van Noort ${ }^{2}$, M. Szydlarski ${ }^{1}$, \\ J. Blanco Rodríguez ${ }^{4}$, P. Barthol ${ }^{2}$, J. C. del Toro Iniesta ${ }^{5}$, A. Gandorfer ${ }^{2}$, L. Gizon ${ }^{2,6}$, J. Hirzberger ${ }^{2}$, M. Knölker ${ }^{7,10}$, \\ V. Martínez Pillet ${ }^{8}$, D. Orozco Suárez ${ }^{5}$, and W. Schmidt ${ }^{9}$ \\ ${ }^{1}$ Institute of Theoretical Astrophysics, University of Oslo, P.O. Box 1029 Blindern, NO-0315 Oslo, Norway; shahin.jafarzadeh@astro.uio.no \\ ${ }^{2}$ Max Planck Institute for Solar System Research, Justus-von-Liebig-Weg 3, D-37077 Göttingen, Germany \\ ${ }^{3}$ School of Space Research, Kyung Hee University, Yongin, Gyeonggi 446-701, Korea \\ ${ }^{4}$ Grupo de Astronomía y Ciencias del Espacio, Universidad de Valencia, E-46980 Paterna, Valencia, Spain \\ 5 Instituto de Astrofísica de Andalucía (CSIC), Apartado de Correos 3004, E-18080 Granada, Spain \\ ${ }^{6}$ Institut für Astrophysik, Georg-August-Universität Göttingen, Friedrich-Hund-Platz 1, D-37077 Göttingen, Germany \\ ${ }^{7}$ High Altitude Observatory, National Center for Atmospheric Research, P.O. Box 3000, Boulder, CO 80307-3000, USA \\ ${ }^{8}$ National Solar Observatory, 3665 Discovery Drive, Boulder, CO 80303, USA \\ ${ }^{9}$ Kiepenheuer-Institut für Sonnenphysik, Schöneckstr. 6, D-79104 Freiburg, Germany \\ Received 2016 August 4; revised 2016 September 14; accepted 2016 October 10; published 2017 March 22
}

\begin{abstract}
A dense forest of slender bright fibrils near a small solar active region is seen in high-quality narrowband Ca II $\mathrm{H}$ images from the SuFI instrument onboard the SUNRISE balloon-borne solar observatory. The orientation of these slender Ca II H fibrils (SCF) overlaps with the magnetic field configuration in the low solar chromosphere derived by magnetostatic extrapolation of the photospheric field observed with SUNRISE/IMaX and SDO/HMI. In addition, many observed SCFs are qualitatively aligned with small-scale loops computed from a novel inversion approach based on best-fit numerical MHD simulation. Such loops are organized in canopy-like arches over quiet areas that differ in height depending on the field strength near their roots.
\end{abstract}

Key words: methods: observational - Sun: chromosphere - Sun: magnetic fields

\section{Introduction}

Large-scale solar magnetism is thought to be generated by dynamo processes in the solar interior (reviews by Choudhuri 2003; Ossendrijver 2003; Charbonneau 2005, 2010). The interior magnetic fields rise through the convection zone driven by their buoyancy, emerge at the surface, and extend through the entire solar atmosphere (Moreno-Insertis 1986; Schüssler et al. 1994; Caligari et al. 1995). They manifest themselves at the photospheric surface on all spatial scales, expand with height, and re-orient with a range of inclination angles depending on their larger-scale configurations and mutual interactions (e.g., Stenflo 1989; Solanki 1993, 2001; Solanki et al. 2006; de Wijn et al. 2009).

In addition, small-scale magnetic fields are thought to be produced by small-scale turbulent dynamo action close to the surface (Vögler \& Schüssler 2007; Danilovic et al. 2010; Stenflo 2012; Rempel 2014; see also Borrero et al. 2015 for a review.)

Magnetic canopies are particular field configurations in which nearly horizontal field lines lie over a region with weaker (or no) field. They are produced when the field in magnetic elements, pores, or sunspots bends over as these features expand. These horizontal field lines can either return to the surface, connecting magnetic surface features of opposite polarities, or expand laterally until they meet same-polarity fields from other surface features (e.g., Gabriel 1976; Jones 1985; Solanki \& Steiner 1990; Bray et al. 1991; review by Wedemeyer-Böhm et al. 2009). Canopies may occur at different atmospheric heights. Estimations range between the mid photosphere and high chromosphere depending on the size and field strength of the concentrations at the surface and/or

\footnotetext{
${ }^{10}$ The National Center for Atmospheric Research is sponsored by the National Science Foundation.
}

the distances between them (e.g., Spruit 1981; Giovanelli \& Jones 1982; Jones \& Giovanelli 1982; Roberts 1990; Buente et al. 1993; Bruls \& Solanki 1995; Solanki et al. 1991; Zhang $\&$ Zhang 2000). A numerical simulation by Rosenthal et al. (2002) suggested canopy heights ranging over $800-1600 \mathrm{~km}$ for network and internetwork areas, with the value depending strongly on the thermodynamic properties of the atmosphere inside and outside the magnetic feature (Solanki $\&$ Steiner 1990).

Direct observation of magnetic fields at chromospheric heights are rather challenging and consequently rare (see Lagg et al. 2015). It is generally believed that the long fibrils visible in the core of $\mathrm{H} \alpha$ wherever there is some magnetic activity are mapping fields overlying quieter internetwork regions (e.g., Wiegelmann et al. 2008) in the form of supergranulation cellcovering canopies. Comparisons between elongated structuring and field topography in the upper chromosphere have suggested that fibrils outline magnetic fields at these heights, from Stokes inversions of the Ca II $854.2 \mathrm{~nm}$ line by de la Cruz Rodríguez \& Socas-Navarro (2011) and Rouppe van der Voort $\&$ de la Cruz Rodríguez (2013), of the He I $1083 \mathrm{~nm}$ line by Schad et al. (2013). The most detailed modeling test of this assumption was done by Leenaarts et al. (2015) using a stateof-the-art MHD simulation, concluding that the synthetic fibrils map field lines fairly well during their start but not at subsequent wave modulation.

Schrijver \& Title (2003) have argued that there should be ubiquitous magnetic loops returning to the surface at much smaller scales than network or supergranulation cells. Such shorter-extent lower-lying canopies in active regions have not been found so far. Although, in the quiet Sun, Wiegelmann et al. $(2010,2013)$ showed the presence of short, low-lying, and highly dynamic loops from magnetic field extrapolations. 
This study addresses this issue using high-quality image sequences from the SUNRISE balloon-borne solar observatory during its second flight (Solanki et al. 2017). The target was a growing active region containing a leading spot (not observed) and a group of pores near disk center. In a high-resolution image sequence obtained with the narrowband $\mathrm{Ca}$ II $\mathrm{H}$ filter in the SUNRISE/SuFI imager we noticed very thin and long bright features, which we call slender $\mathrm{Ca}$ II $\mathrm{H}$ fibrils (SCF) henceforth. They appear to emanate from the more active areas in the field of view that contain pores and dense small strong-field magnetic concentrations. The physical properties of these SCFs have been investigated by Gafeira et al. (2017b). In addition, Jafarzadeh et al. (2017) and Gafeira et al. (2017a) studied transverse and sausage-mode oscillations in the SUNRISE/ SuFI SCFs and provided propagation speeds of both types of waves.

The on-disk SCFs represent a rather new phenomenon. Similar fibrils were seen earlier in high-resolution Ca II K and $\mathrm{Ca}$ II $\mathrm{H}$ images from the Swedish $1 \mathrm{~m}$ Solar Telescope (Pietarila et al. 2009; Henriques \& Kiselman 2013) and in SUNRISE-II Mg II k and Ca II H images (Riethmüller et al. 2013; Danilovic et al. 2014). We note that, although from the same instrument and the same $\mathrm{CaIIH}$ passband, the data set under study samples a different solar region and is of higher quality than those shown by Riethmüller et al. (2013) and Danilovic et al. (2014; i.e., they were treated by an improved image-reconstruction technique).

Comparable long slender fibrils were also observed near the solar limb in Ca II H images from the Dutch Open Telescope and called "straws" by Rutten (2006). These were then equated to off-limb spicules-II discovered in $\mathrm{Ca}$ II $\mathrm{H}$ image sequences with Hinode and identified as Alvénic-wave phenomena by De Pontieu et al. (2007) with torsion added by De Pontieu et al. (2012). Their on-disk counterpart in $\mathrm{H} \alpha$ takes the form of rapid blue-wing excursions (RBE, Langangen et al. 2008; Rouppe van der Voort et al. 2009; Sekse et al. 2012) and similar rapid red-wing excursions (RRE, Sekse et al. 2013). Their drivers remain unidentified (Pereira et al. 2012).

The SUNRISE SCFs differ from such spicule-II straws because they are found near disk center and are extremely thin. It would be of much interest to compare them to RBEs and RREs in $\mathrm{H} \alpha$ and also to compare them with the onsets of the longer and wider fibrils observed in the core of $\mathrm{H} \alpha$. Unfortunately, there were no simultaneous high-resolution observations in these lines of the SUNRISE target, so we cannot study the question whether, and if so how, the SUNRISE SCFs relate to spicules-II and/or long $\mathrm{H} \alpha$ fibrils.

On the other hand, the SUNRISE observations do provide high-quality solar-surface magnetograms from the IMaX instrument (Martínez Pillet et al. 2011), while modern field extrapolation techniques (e.g., Wiegelmann et al. 2015) and yet newer data-constrained MHD simulation techniques (Riethmüller et al. 2017) permit trustworthy estimation of the actual field configuration above the surface from these magnetograms. We employ these techniques here to test the premise that the observed SCFs are field-aligned and may therefore serve to establish the magnetic configuration above the surface. We concentrate in particular on the question what height these fibril-marked fields reach.

The organization of this publication is as follows. In the next section, we use a well-documented state-of-the-art numerical MHD simulation to study magnetic configurations at multiple heights. In Section 3 we detail the SUNRISE observations. In Section 4 we compare these to magnetic field extrapolations and MHD simulations defined by the IMaX magnetograms. We end the study with a brief conclusion.

\section{Field Configuration in a Bifrost Simulation}

In order to illustrate magnetic configurations located at multiple heights from the photosphere to the corona we visualize the geometry of magnetic field lines in a 3D snapshot from the well-documented state-of-the-art simulation with the $3 \mathrm{D}$ radiative-MHD code Bifrost (Gudiksen et al. 2011) that has been described and made public by Carlsson et al. (2016), to which we refer for more detail. The same simulation was used by Leenaarts et al. (2015). In brief, the Bifrost code solves the MHD equations on a staggered grid and includes radiation by solving the radiative transfer equations along many rays using a short-characteristic method and multi-group opacities (Nordlund 1982; Hayek et al. 2010). The chromospheric radiative losses are computed in non-LTE and thermal conditions at high temperatures are taken into account. In this simulation, the hydrogen ionization balance was computed including nonequilibrium retardation.

The simulation snapshot used here is dominated by two opposite-polarity enhanced network patches and samples the entire atmosphere in a volume of $24 \times 24 \mathrm{Mm}^{2}$ horizontally (with $504 \times 504$ grid points giving $48 \mathrm{~km}$ resolution), extending from $2.4 \mathrm{Mm}$ below the visible surface (defined as optical depth unity at $500 \mathrm{~nm}$ ) to $14.4 \mathrm{Mm}$ above it. The vertical dimension is spanned by 496 grid points with variable sizes changing from $19 \mathrm{~km}$ up to a height of $5 \mathrm{Mm}$ from the bottom of the computational box and then increasing to $100 \mathrm{~km}$ close to the top boundary. We limit the vertical dimension to $14 \mathrm{Mm}$ in our displays here (see below). The maximum field strength has an absolute value of $1.9 \mathrm{kG}$ at the visible solar surface.

To visualize the magnetic configuration we display field lines through the entire simulation cube that pass through specific $(x, y, z)$ point locations, which are randomly positioned through a layer at a selected height. The number of these starting points for field-line tracing is about 2000 and is kept small for better visibility. A tracing algorithm then produces field lines following the vector field from these initial starting points in both directions until they meet a boundary surface of the simulation volume. This algorithm so fills the simulation cube with field lines that chart fields over a wide range of strengths and configurations, with the common property that they all pass through the selected layer and do so at random locations.

Selected results are shown in Figure 1. Panel (a) has the starting layer at the surface so that the traced field lines sample all fields in the simulation volume. We note that all fields in the simulation box originate below the surface, i.e., there are no sagging loops coming into the box from the top or the sides. By putting the selective starting layer higher, in the low corona, transition region/upper chromosphere, and low chromosphere respectively, the partial corresponding field configurations are mapped in panels (b)-(d). For better visibility, the field lines in panel (d) are cut at height $1400 \mathrm{~km}$. The selected starting layers in panels (a)-(d) are marked with the horizontal dashed lines and range between, $0-100 \mathrm{~km}, 5000-7000 \mathrm{~km}, 1800-2800$, and 500-1000 km, respectively. We comment that fewer highlying field lines are seen in panel (c) compared to those in panel (b) because fewer of them are caught when the number of 
(a)

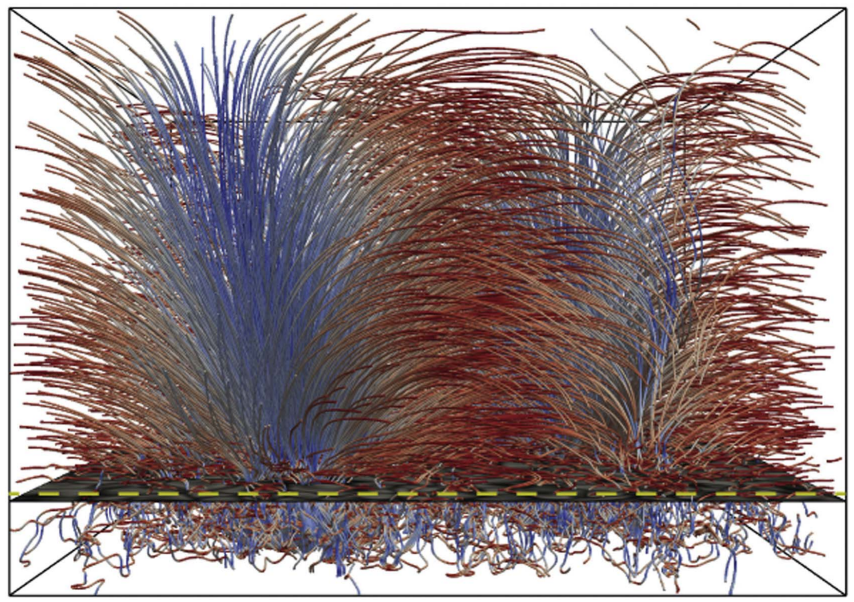

(c)

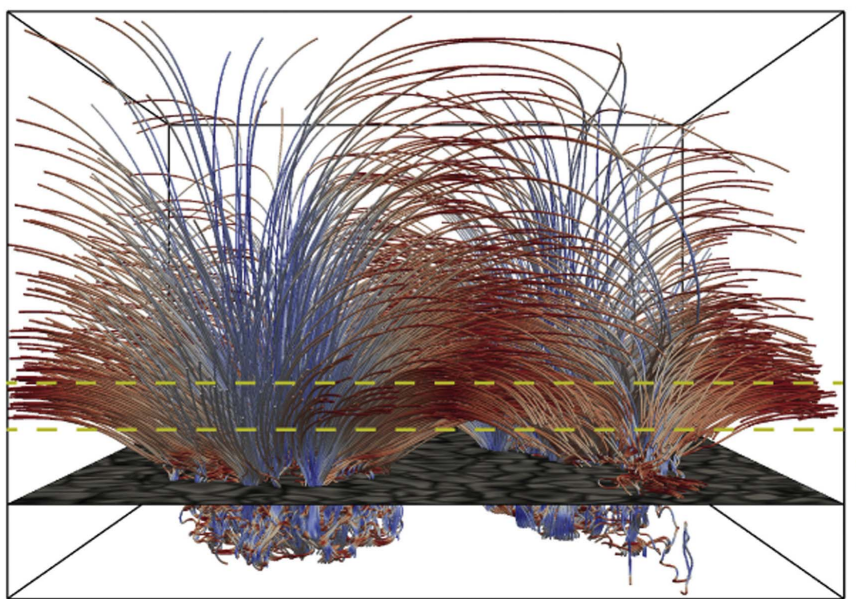

(b)

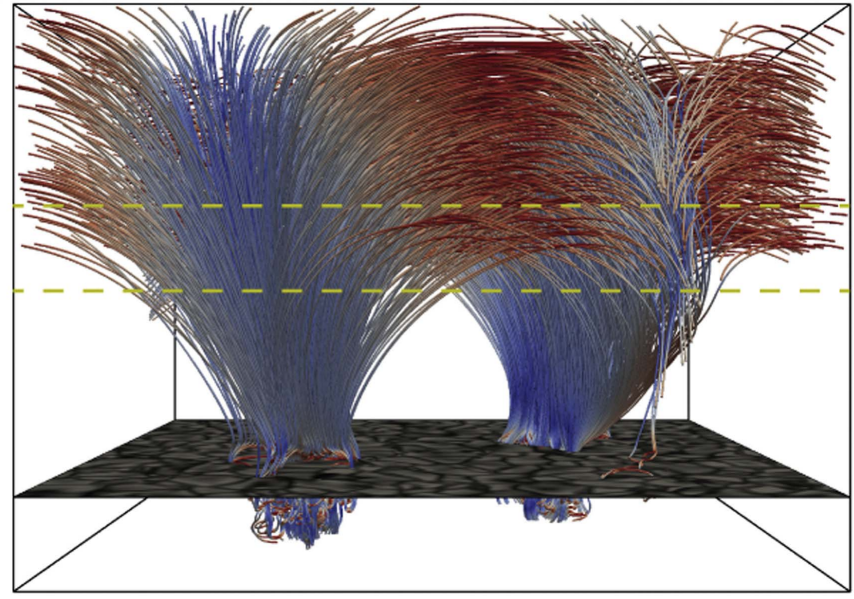

(d)

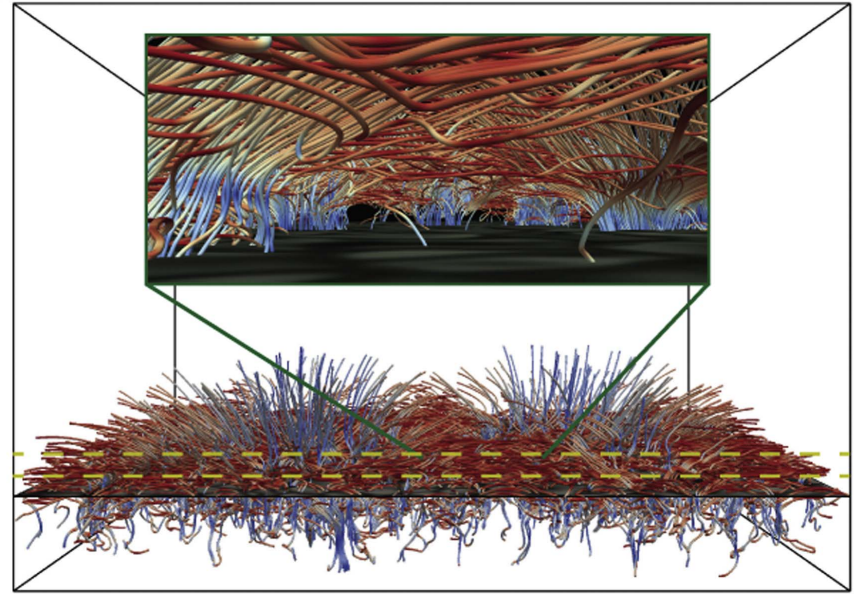

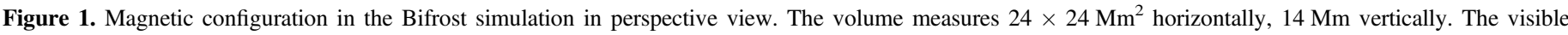

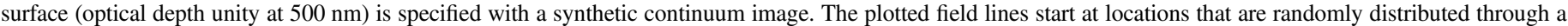

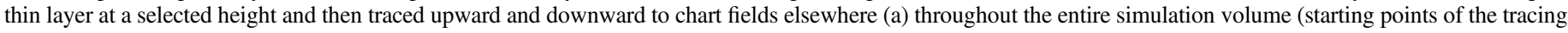

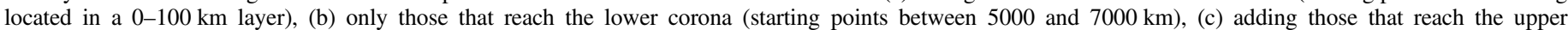

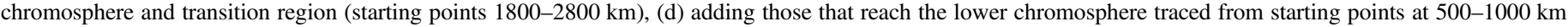

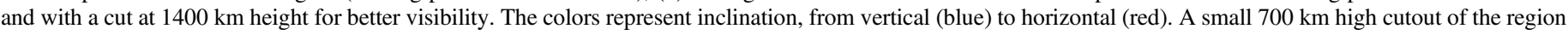
between the two main footpoints is magnified in the inset in (d).

traced field lines is fixed and we start deeper down in the atmosphere.

The height reached by a single field line in Figure 1 is usually higher when the field is stronger in the root where it passes through the surface. As a result, the figure suggests the presence of a particular type of magnetic canopy, if we consider fields originating at the surface in a particular type of feature. Depending on the type of footpoint, the height of its lower boundary surface can be quite different. For example, panel (b) displays a large volume relatively free of field extending to large height between and around the two network patches, with many patch-connecting field lines above it. This is because most field lines in panel (b), which selects only highreaching ones, are rooted in the two patches of strong-field opposite-polarity network to gain such height. The arch they span above the quiet area between the network patches represents a high canopy. This is not a canopy with field-free or high- $\beta$ plasma underneath as in the more common definitions of magnetic canopy (Solanki \& Steiner 1990), or in the canopy definition as sound-speed and Alfvén-speed equality of Bogdan et al. (2003), but as a dome having unplotted fields inside that originate in less strong sources and therefore do not reach as high.

Similarly, the inset in panel (d) shows a small interior part of the volume that illustrates a low and smaller canopy of this type between the two network areas that remains within the low chromosphere. It implies that almost all field lines passing through $500 \mathrm{~km}$ height connect to stronger surface fields closer to the network patches (blue stalks to the side) and do not connect the quiet surface containing mainly weak fields separating the strong opposite-polarity patches.

The importance of this display format showing root-strength canopies with different heights is that they indicate connectivity for actual observed feature canopies to properties of their source regions. For example, if the observed canopies outlined by long $\mathrm{H} \alpha$ fibrils reach a specific height or have a 
characteristic minimum height, then the suggestion from this simulation rendering is that the mechanism in the fibril root region that produces the observed fibrils must operate at a specific field strength, or only at values above a particular field strength.

The conclusion from this demonstration is that the existence of many small low-lying loops in internetwork regions postulated by Schrijver \& Title (2003) is supported by the Bifrost simulation, but that it also suggests that there is a hierarchy of canopy-like arched field configurations, which map field strengths near the field roots at the surface, and that the height of these arches increases with higher field strengths at the roots, or at least at one of the roots (see Wiegelmann et al. 2010).

\section{Observations from SUNRISE}

\subsection{Observations and Reduction}

The primary data set used here was recorded with the narrowband $\mathrm{Ca}$ II $\mathrm{H}$ filter (with $\mathrm{FWHM} \approx 0.11 \mathrm{~nm}$ ) of the SUNRISE Filter Imager (SuFI; Gandorfer et al. 2011) onboard the 1-meter SUNRISE balloon-borne solar observatory (Solanki et al. 2010; Barthol et al. 2011; Berkefeld et al. 2011) during its second flight in 2013 (SUNRISE-II; Solanki et al. 2017). Our seeing-free image sequence with high spatial and temporal resolution was collected between 23:39 UT on 2013 June 12 and 00:38 UT on 2013 June 13. The Ca II H images cover a field of view $(\mathrm{FOV})$ of $(15 \times 38) \operatorname{arcsec}^{2}$ that covered part of the following polarity magnetic features of NOAA AR 11768 (including a few small pores and plages) near solar disc center (cosine of the heliocentric angle $\mu \approx 0.93$ ). These high-quality data were corrected for wavefront aberrations by multi-frame blind deconvolution (MFBD; van Noort et al. 2005).

We note that several other $\mathrm{Ca} I \mathrm{H}$ image sequences of various solar regions were obtained during the SUNRISE observations from 2013 June 12 to 17 (Solanki et al. 2017). For comparison with photospheric structures, we also use images recorded with the SUNRISE/SuFI $300 \mathrm{~nm}$ filter. In addition, full-Stokes $(I, Q, U$, and $V)$ images in the magnetically sensitive line Fe I $525.02 \mathrm{~nm}$ were obtained with the SUNRISE Imaging Magnetograph eXperiment (IMaX; Martínez Pillet et al. 2011).

We use the magnetograms obtained from the SUNRISE/ IMaX images to extrapolate the magnetic field into the solar chromosphere. These extrapolations are based on magnetohydrostatic equilibria and have been described by Wiegelmann et al. (2017). They also employ wider FOV but lower resolution and lower sensitivity SDO/HMI data (Pesnell et al. 2012; Schou et al. 2012). An earlier application of the magnetohydrostatic equilibrium technique to quiet-Sun observations from the 2009 flight of SUNRISE (SUNRISE-I) was made by Wiegelmann et al. (2015), who also gave a more detailed description of the employed method.

We also compared our $\mathrm{Ca}$ II $\mathrm{H}$ images with co-aligned observations in $\mathrm{He}$ II $30.4 \mathrm{~nm}$ from SDO/AIA (Lemen et al. 2012).

\subsection{SUNRISE Results}

Slender $\mathrm{Ca}$ II $\mathrm{H}$ fibrils-Figure 2(a) shows a Ca II H image after sharpening with an unsharp-mask filter to gain better visibility of the slender fibrils. The SCFs in this sequence were identified by restoring the $\mathrm{Ca} \mathrm{II} \mathrm{H}$ images in a multi-step procedure described in detail by Jafarzadeh et al. (2017). In short, the inhomogeneities in the images due to non-uniform background solar intensity, noise, and geometric distortions are minimized using a real-space spatial bandpass filter (Jafarzadeh et al. 2013) and the SCFs are modeled as bright elongated structures in the restored images.

Figure 2(b) overplots the detected SCFs corresponding to the first panel superimposed on a low-contrast version of the cospatial and co-temporal $300 \mathrm{~nm}$ wider-band image. Figure 2(c) displays this wide-band image at full contrast.

The corresponding Stokes $V$ map from IMaX is plotted in Figure 2(d). It is an average over four wavelength positions (at $\pm 0.008 \mathrm{~nm}$ and $\pm 0.004 \mathrm{~nm}$ from the Fe I $525.02 \mathrm{~nm}$ line core) that was re-scaled and co-aligned with the SuFI images in panels (a)-(c). The red ellipses in Figures 2(c) and (d) mark two areas with fairly dense network field concentrations from which many of the fibrils emanate outward with varying lengths, although many fibrils start already to the left of these features (as seen in the figure), seeming to come from the large pore at the edge of the FOV. The yellow rectangles outline the quietest areas in the SuFI FOV. The FOV of the images shown in Figure 2 is vertically flipped and slightly rotated with respect to the true orientation on the Sun. For the correct orientation, see Solanki et al. (2017).

We now list the main SCF properties noted from these observations.

1. There are relatively short SCFs radiating out from the large pore near the mid-left edge of the images. Long SCFs fanning out from this pore are also observed. The fibrils emanating away from the smaller pores near the bottom-right corner of Figure 2(b) are shorter than those from the large pore. These are among the shortest in our image sequence.

2. Many long SCFs emanate from the plage near the pores. These plage regions are mostly concentrated in areas to the right side of the large pore and the lower left side of the small pores that are marked by red ellipses in Figure 2. While the fibrils starting in the former extend mostly close to the $x$ direction, the latter are more oriented in the $y$ direction. A few short SCFs with other angles are also observed in both areas.

3. Inspection of SCF endings on the photospheric $300 \mathrm{~nm}$ images shows that some SCFs seem to have one or both footpoints rooted in magnetic field concentrations, whereas many others seem to be located at granules. The SCFs emanating from the pores seem to always have one footpoint in the umbra. However, we stress that such footpoint identification is rather uncertain.

4. The two quiet areas marked by yellow boxes show some SCFs but these mostly extend from neighboring magnetic patches.

Height of formation-We now turn to the question of whether these observations in themselves permit estimation of SCF formation heights. In order to obtain an initial estimate, we have computed line-depression contribution functions (CF) for the narrowband SuFI Ca II H filter using the $1 \mathrm{D}$ version of the RH code of Uitenbroek (2001), which solves the radiative transfer and statistical equilibrium equations in non-LTE and includes partial redistribution for a given 1D model atmosphere. This computation is similar to the one reported in Danilovic et al. (2014). Our motivation to include and present 
(a)

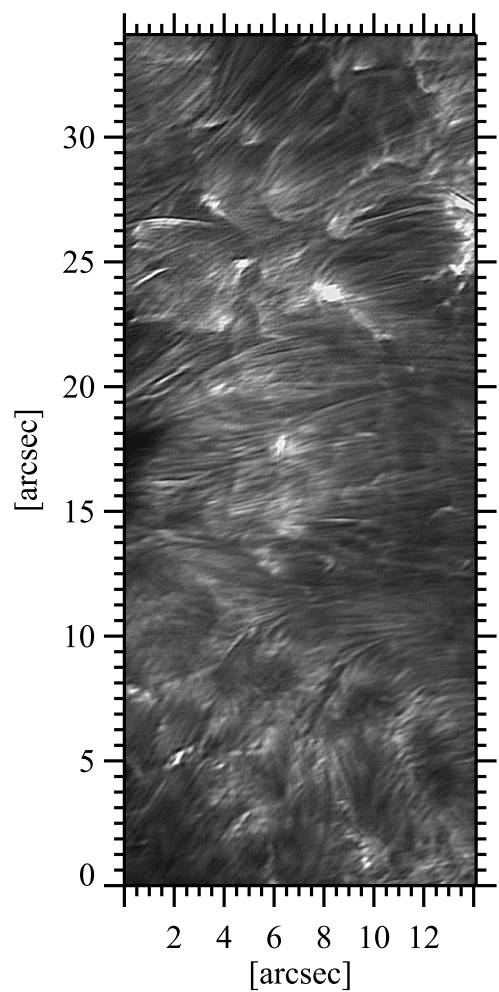

(b)

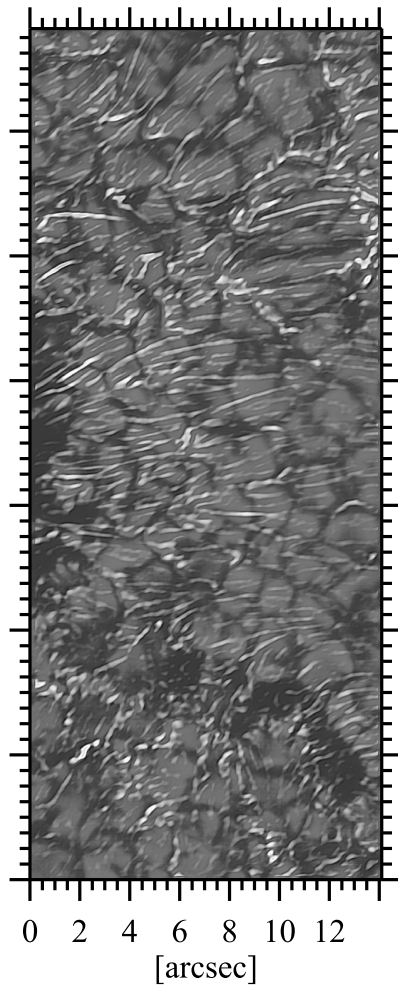

(c)

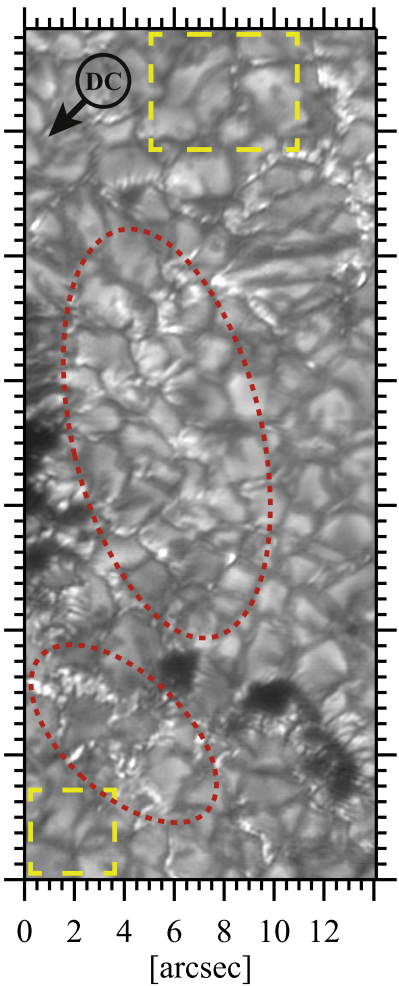

(d)

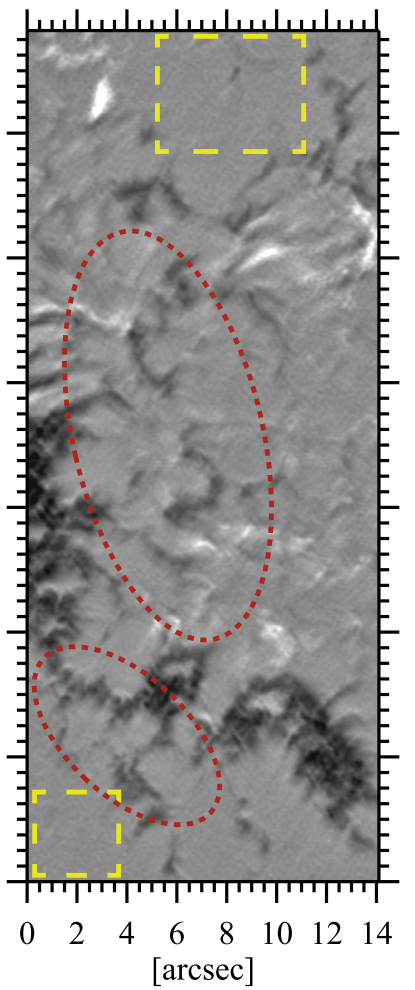

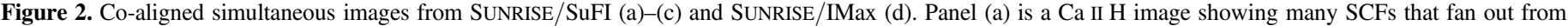

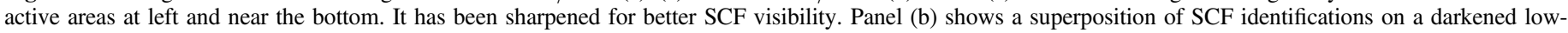

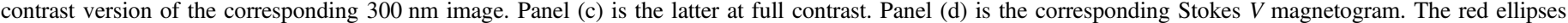
outline two areas with many magnetic concentrations and the yellow boxes two quiet areas. The direction to disk center is specified in panel (c).

these rather simplistic estimates ignoring the actual fine structure of the solar atmosphere is that they already suggest a bimodal response that likely also affects our observations.

We used three different models from Fontenla et al. (1993), FALC, FALF, and FALP, that were constructed to represent average quiet Sun, bright network, and plage, respectively. Figure 3 shows the resulting synthetic $\mathrm{Ca}$ II $\mathrm{H}$ profiles, their multiplication with the transmission profile of the SuFI Ca II H filter, and the corresponding CFs for the three models with average formation heights. The CFs for FALC and FALF obtain their largest contributions from the photosphere below $550 \mathrm{~km}$; only FALP predicts a larger fraction $(\approx 60 \%)$ from higher layers. This difference is already evident in the synthetic profiles in which the $\mathrm{H}_{2 V}$ and $\mathrm{H}_{2 R}$ emission peaks are highest for the plage model, presumably portraying magnetic heating (e.g., Linsky \& Avrett 1970; Skumanich et al. 1975; Ayres et al. 1986; Solanki et al. 1991; Fontenla et al. 2009). Note, however, that the heights obtained from Figure 3 may be overestimated, as the narrowband $\mathrm{Ca}$ II $\mathrm{H}$ filter of SuFI may have been somewhat offset relative to the line core, as noted by Solanki et al. (2017).

These standard-model estimates may nonetheless serve to interpret the background scenes in Figure 2. We first discuss the quietest areas outlined by the yellow boxes. For comparison, Figure 4 shows a truly quiet area taken with the Ca II H filter on 2013 June 13 at 12:53 UT. We comment that these quiet-Sun images were phase diversity reconstructed employing averaged wavefront errors (see Hirzberger et al. 2010, 2011) and were not processed by MFBD. Higher resolution of such images from the SUNRISE flight of 2009 are shown by Solanki et al. (2010). The quiet-Sun scene in Figure 4 clearly contains no chromospheric features but only reversed granulation, wave interference patterns including $\mathrm{H}_{2 V}$ bright points, and a few magnetic bright points. The second panel shows the simultaneous wide-band granulation image but with intensity reversed so that the now bright intergranular lane pattern can directly be compared to the cellular ridge patterns in the first panel. This shows that the narrowband scene is not simply dominated by granulation reversal but also contains significant other components. A similar study by Rutten et al. (2004) showed that these mostly represent acoustic wave interference, possibly also gravity-wave interference. The upshot is that the background patterns represent formation in the left-hand CF peaks of Figure 3, i.e., in the upper photosphere, and that the SuFI SCFs lie above this height.

Comparison with He II $30.4 \mathrm{~nm}$-In order to search for relationships between the SCFs and comparable elongated fibrilar structures in the upper atmosphere we have compared our $\mathrm{Ca}$ II $\mathrm{H}$ image sequence with corresponding co-temporal and co-aligned image sequences recorded in He II $30.4 \mathrm{~nm}$ by $\mathrm{SDO} / \mathrm{AIA}$. We take the latter as a proxy for $\mathrm{H} \alpha$ fibrils since we have no simultaneous observations in the latter line and, at least in active regions, there tends to be general correspondence between fibrils seen in He II $30.4 \mathrm{~nm}$ and in $\mathrm{H} \alpha$ (e.g., the IBIS mosaic of Reardon \& Cauzzi 2012; Figure 1 of Rutten et al. 2015).

However, in our inspections we found no clear one-to-one correspondence of features in our $\mathrm{Ca}$ II $\mathrm{H}$ filtergrams to the structures in the He II $30.4 \mathrm{~nm}$ images, neither at the same time nor with a time delay. However, even if some of the SCFs (i.e., 

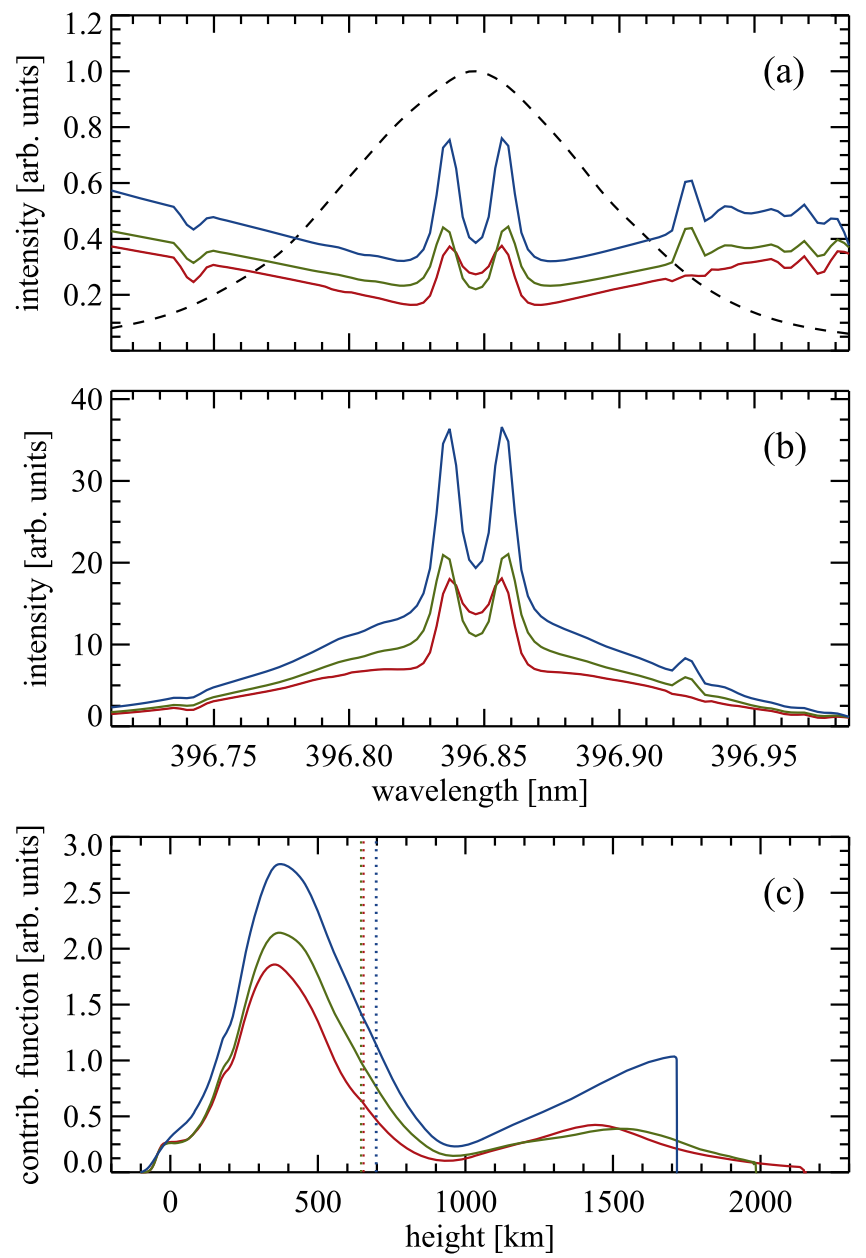

Figure 3. Ca II H computed from FALC (quiet Sun, red), FALF (network, green), and FALP (plage, blue). Panel (a): emergent-intensity spectra. The dashed curve is the transmission profile of the SUNRISE/SuFI Ca II H filter. Panel (b): filter-transmitted intensity spectra. Panel (c): contribution functions for the summed transmitted intensities. The vertical lines mark the first-moment height-of-formation averages.

the longer and thicker ones) have counterparts in the hotter He II $30.4 \mathrm{~nm}$ line, the spatial resolution of the AIA images is too low to show very thin fine structures, such as the SCFs in our SuFI images.

\section{Observation-driven Field Modeling}

\subsection{Magnetic Field Extrapolation using a Magnetostatic Approach}

We now discuss the 3D magnetic configuration above the observed solar region that is obtained from magnetostatic extrapolation of the field into the chromosphere. The theory of the used special class of magnetostatic equilibria has been developed in Low (1991) and applied to the quiet Sun in Wiegelmann et al. (2015). In this approach, the high spatial resolution SUNRISE/IMaX vector magnetogram is embedded into a large-scale and flux-balanced magnetogram of the whole active region observed by SDO/HMI. The composite serves as a boundary condition for the magnetostatic modeling, which yields the field configuration in the non-force-free layers between the photosphere and the mid-chromosphere more reliably than force-free extrapolations, which are limited to the
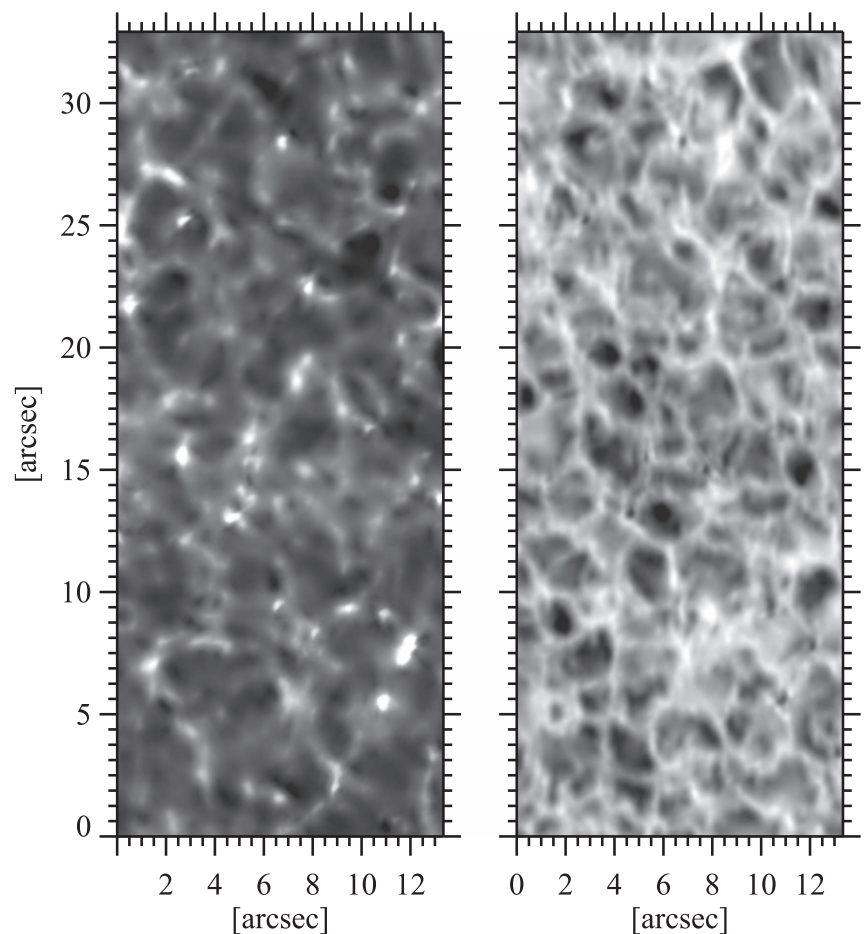

Figure 4. Left: quiet area in the narrow SuFI Ca II H passband. Right: corresponding granulation image in the wide $300 \mathrm{~nm}$ passband with reversed contrast.

low plasma- $\beta$ solar corona. The original computational box has an FOV of $86 \times 86 \mathrm{Mm}^{2}$ and extends through the solar atmosphere up to a height of $8 \mathrm{Mm}$ (with 200 grid points in the vertical direction). Deviations from force-free fields occur mainly below $2 \mathrm{Mm}$ in the mixed plasma- $\beta$ upper photosphere and chromosphere. For more detail, see Wiegelmann et al. (2017).

We limit our displays to the FOV of the SUNRISE/IMaX images and to a height of up to only $1400 \mathrm{~km}$ for better visualization. As in Section 2, we trace and plot field lines passing through starting locations that were selected randomly, in this case within the subvolume above $700 \mathrm{~km}$, i.e., spanning the $700-1400 \mathrm{~km}$ height range. This selection implies that only field lines reaching heights of $700 \mathrm{~km}$ or higher are shown, i.e., chromospheric fields. One reason for restricting ourselves to these field lines is because (as we saw in Section 2) fields peaking lower issue from weaker fields at the solar surface that are more strongly affected by noise in the IMaX data. Hence these higher fields are more reliably extrapolated.

Figure 5(a) shows this selective field extrapolation at the moment the images plotted in Figure 2 were recorded. The FOV of the SuFI Ca II H images is marked by the black rectangle. Many field lines form low-lying, rather flat loops that close within the FOV (i.e., those with blue/green at both ends, and long red parts in the middle; examples are field lines shown near the mid-right edge of the FOV of the SuFI images). Some other field lines go straight up (such as those fanning out from the pores, e.g., near the bottom of the SuFI FOV, or outside the SuFI FOV on its mid-to-upper left side), while others remain flat for some distance and then go out of the FOV (unipolar canopy type; examples are the field lines depicted in the upper left and in the lower-right corners of the box). Many other filed lines pass in and out of the displayed FOV at the sides (e.g., those shown close to the lower left corner). For 
Inclination angle $[\mathrm{deg}]$
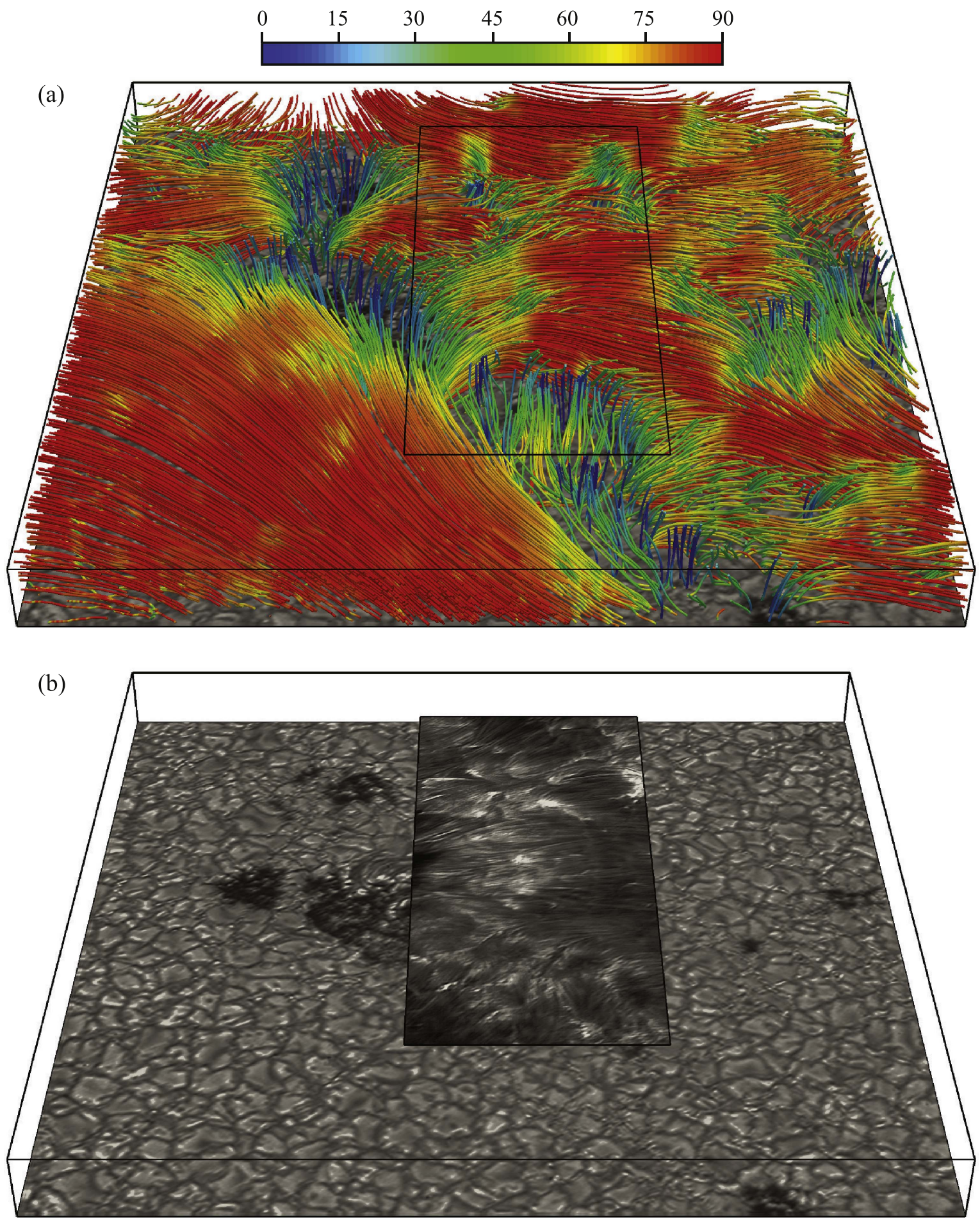

Figure 5. Magnetostatic extrapolation of the surface magnetic field observed with IMaX and SDO with height. In panel (a) the rectangle specifies the field of view of the SuFI Ca II H image; the colors specify inclination angles. Panel (b) shows the corresponding observed IMaX continuum image and SuFI Ca II H image. The boxes measure $37 \times 37 \mathrm{Mm}^{2}$ horizontally and $1400 \mathrm{~km}$ vertically, with vertical scale doubling for better visibility.

reference, the IMaX continuum image and the $\mathrm{Ca} I \mathrm{H}$ filtergram are depicted in Figure 5(b). The figure covers the full IMaX FOV rather than only the small SuFI FOV to permit inspection of the surrounding field configuration. Comparison of the two panels in Figure 5 indicates that the majority of the SCFs are oriented in the same directions as the field lines within the SuFI FOV. There are, however, disagreements in the lower left corner and near the upper edge of the $\mathrm{Ca}$ II $\mathrm{H}$ image. These are the quietest regions in the SuFI images (outlined by yellow frames in panels (c) and (d) of Figure 2) and sample only low atmospheric heights. Although the extrapolation does contain chromospheric field lines above these areas there are no SCF-like features with sufficient opacity for visibility. 


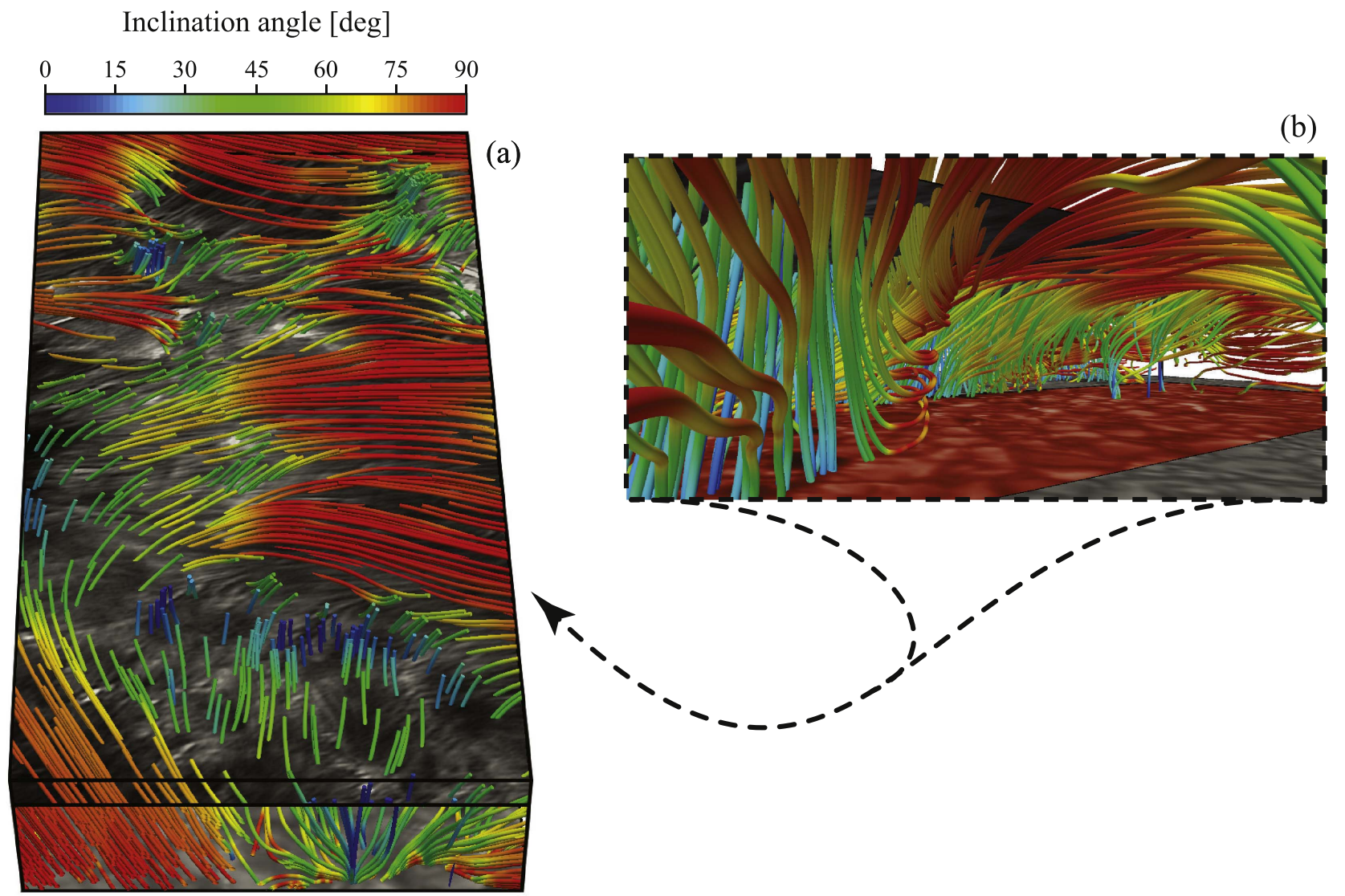

Figure 6. Panel (a): same as Figure 5(a), but limited to the SuFI FOV and with the Ca II H image inserted at 1000 km height. Panel (b) gives an inside view of the apparent canopy from a viewing angle shown by the arrow pointing to panel (a). The SuFI FOV is indicated by red color on the IMaX continuum intensity image in panel (b).

Overall, the comparison suggests that the majority of the SCFs do outline magnetic fields, but with some exceptions.

Field inclinations should carefully be taken into account in this comparison, since a more vertical field produces shorter SCFs within the $\mathrm{Ca} I \mathrm{H}$ response range in height. Thus, the presence of relatively long SCFs in the center-right part of the FOV (right-hand part of the larger ellipse in Figure 2) agrees with the presence of long field lines lying nearly horizontally in this area, while shorter SCFs elsewhere indeed correspond to less horizontal field lines.

Figure 6(a) is similar to Figure 5(a), but limited to the FOV of SuFI and with the Ca II H image inserted at $1000 \mathrm{~km}$ height. This facilitates the comparison of the field lines with different inclination angles and the SCFs in the Ca II H image. A small part of the field configuration in Figure 6(a) is shown enlarged as a 2D cut in Figure 6(b) from inside the field configuration along a line of sight, indicated by the arrow in Figure 6(a). The SuFI FOV is indicated by red-coloring the IMaX continuum image. It illustrates the canopy-like dome made by those field lines that make it to $700 \mathrm{~km}$ or higher and are mostly rooted in the pore and plage field concentrations.

We comment that these extrapolations are probably missing low-lying fields, because due to the higher noise of the IMaX observations on SUNRISE-II relative to SUNRISE-I many weakfield features are missing. From the work of Wiegelmann et al. (2010, 2013), as well as from our study described in Section 2, we know that these weak (small-flux) features lie at the footpoints of low-lying loops. Alternatively, the low-lying loops are not well modeled because of, e.g., limitations of the magnetostatic equilibrium.

The conclusion of this section is that the orientation of the majority of the observed SCFs corresponds well to the field configuration in the low chromosphere as derived from the IMaX magnetograms. However, a small fraction does not map the extrapolated field lines. The latter cases are mostly located in less-organized areas.

\subsection{MHD-assisted Stokes Inversion}

In addition to our inspection of the magnetic configuration determined from observed-field extrapolation, we similarly investigate the photospheric field geometry with a novel MHDassisted Stokes inversion technique (MASI; Riethmüller et al. 2017). In this approach, an initial MHD simulation carried out with the MURaM code (Vögler et al. 2005) containing similar features as in the observed area is used to obtain the 1D atmospheric models providing the best-fit synthetic profiles to the Stokes profiles observed by IMaX in each pixel. This is done by reshuffling the simulation columns to best match the observations and then rerunning the simulation to get a physically consistent result. After multiple iterations this technique delivers a modified MHD simulation starting from a magnetic geometry and thermodynamic structure similar to that of the observation. After a simulation run of about 30 minutes solar time to reach relaxation, a physically consistent MHD simulation is obtained which 
Inclination angle $[\mathrm{deg}]$
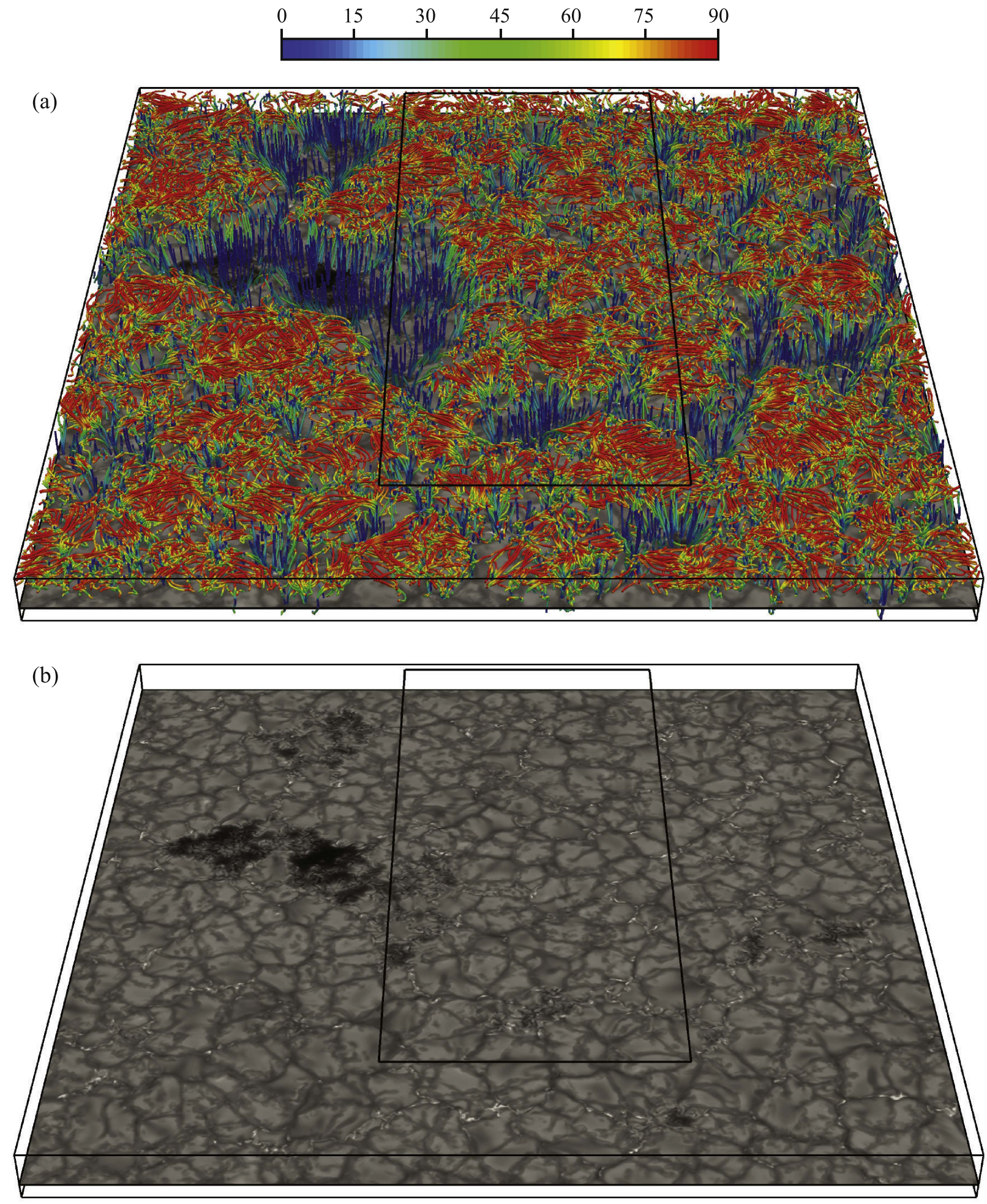

Figure 7. Magnetic field configuration in the solar photosphere from a novel MHD-assisted inversion technique (panel a). The colors specify inclination angles. The synthetic image in panel (b) represents a target similar to the IMaX observation in Figure 5(b). An area with the size of the SuFI Ca II H images is indicated in both panels with a rectangle. The volume measures $34 \times 34 \mathrm{Mm}^{2}$ horizontally and $1000 \mathrm{~km}$ vertically (of which $700 \mathrm{~km}$ lie above the continuum intensity level). The vertical scales are doubled for better visibility.

closely resembles the observation. For more details see Riethmüller et al. (2017).

We took such a resulting MASI cube and plot in Figure 7 the field configuration in a similar manner as in Figures 1, 5 and 6.
It represents heights below $700 \mathrm{~km}$ on average from the solar surface. The area measures $34 \times 34 \mathrm{Mm}^{2}$, similar to (but slightly smaller than) the IMaX FOV; the resolution is $20.8 \mathrm{~km} /$ pixel. Field lines were again traced starting from 
random points in a layer reaching from the top boundary to $350 \mathrm{~km}$ below it. A continuum intensity image is added in panel (b) for reference. We note that these simulations are based on the assumption of LTE and are limited to photospheric heights. Therefore, the field configuration from MASI does not contain chromospheric structures as the ones defining the majority of our SCFs. Hence, Figure 7(a) represents only photospheric field lines for an FOV similar to that in our observation. The black rectangle indicates a simulation area with the same size and similar content as our SuFI FOV. It shows many small loops in the upper photosphere. These small loops are likely peaking from weaker fields at the solar surface (as we saw in Section 2) compared to the extrapolated fields illustrated in Figures 5 and 6. Such photospheric loops could not be reliably extrapolated because the weaker fields are more strongly affected by noise in the IMaX data. Many of the small low-lying loops shown in Figure 7(a) display a preferred orientation in the $x$ direction, which agrees well with the observed SCFs and the field lines at a somewhat greater height in the magnetostatic extrapolation. Although this simulation result and the observation are not comparable on a one-to-one basis, we speculate that such low short loops may represent some of the shorter observed SCFs. Remember that the passband of the SuFI Ca II H filter yields a wide contribution range from photosphere to chromosphere (Section 3), so that some of the fibrils may indeed be lying at or slightly below the temperature minimum height. However, they cannot lie too low, as no such fibrillar structures are visible in the line core of the IMaX line, Fe I $525.02 \mathrm{~nm}$.

The upshot from this modeling together with the magnetostatic extrapolation is, that the observed SCFs map field lines in the low chromosphere, around 700-1000 km height.

\section{Conclusion}

The SCFs in our Ca II H images suggest the existence of small-scale heating events in or near plage and network field concentrations that produce thin long signatures in the $\mathrm{Ca} \mathrm{II} \mathrm{H}$ line that become visible at the bandpass and high angular resolution of SUNRISE/SuFI.

Our detailed field extrapolation and MHD-simulationassisted field modeling both suggest that the majority of these features are aligned with and chart magnetic field lines in the low solar chromosphere at a height of around 700-1000 km. Such field lines constitute a low canopy-like dome across the neighboring quiet areas. MHD simulations suggest that such a dome is defined by the field strength where the field lines forming it originate. Areas with less well-organized fields seem to have less good alignment between field elongated structures in $\mathrm{Ca}$ II $\mathrm{H}$.

Future studies should address the nature of these heating events and their relation to comparable phenomena as spiculesII and long $\mathrm{H} \alpha$ fibrils, but these then require simultaneous $\mathrm{H} \alpha$ and $\mathrm{Ca}$ II H (or $\mathrm{Ca}$ II K) observations of at least the quality shown here.

The German contribution to SUNRISE and its reflight was funded by the Max Planck Foundation, the Strategic Innovations Fund of the President of the Max Planck Society (MPG), DLR, and private donations by supporting members of the Max Planck Society, which is gratefully acknowledged. The Spanish contribution was funded by the Ministerio de Economía y Competitividad under Projects ESP2013-47349-C6 and ESP2014-56169-C6, partially using European FEDER funds. The HAO contribution was partly funded through NASA grant number NNX13AE95G. This work was partly supported by the BK21 plus program through the National Research Foundation (NRF) funded by the Ministry of Education of Korea. S.J. receives support from the Research Council of Norway. T.W. acknowledges support by DFG-grant WI 3211/4-1. M.S. acknowledges support from the European Research Council under the European Union's Seventh Framework Programme / ERC Grant agreement nr. 291058. The National Solar Observatory (NSO) is operated by the Association of Universities for Research in Astronomy (AURA) Inc. under a cooperative agreement with the National Science Foundation.

\section{References}

Ayres, T. R., Testerman, L., \& Brault, J. W. 1986, ApJ, 304, 542 Barthol, P., Gandorfer, A., Solanki, S. K., et al. 2011, SoPh, 268, Berkefeld, T., Schmidt, W., Soltau, D., et al. 2011, SoPh, 268, 103 Bogdan, T. J., Carlsson, M., Hansteen, V. H., et al. 2003, ApJ, 599, 626 Borrero, J. M., Jafarzadeh, S., Schüssler, M., \& Solanki, S. K. 2015, SSRv, in press https://doi.org/10.1007/s11214-015-0204-5

Bray, R. J., Cram, L. E., Durrant, C. J., \& Loughhead, R. E. 1991, Plasma Loops in the Solar Corona (Cambridge: Cambridge Univ. Press)

Bruls, J. H. M. J., \& Solanki, S. K. 1995, A\&A, 293, 240

Buente, M., Solanki, S. K., \& Steiner, O. 1993, A\&A, 268, 736

Caligari, P., Moreno-Insertis, F., \& Schüssler, M. 1995, ApJ, 441, 886

Carlsson, M., Hansteen, V. H., Gudiksen, B. V., Leenaarts, J., \& De Pontieu, B. 2016, A\&A, 585, A4

Charbonneau, P. 2005, LRSP, 2, 2

Charbonneau, P. 2010, LRSP, 7, 2

Choudhuri, A. R. 2003, in Dynamic Sun, ed. B. N. Dwivedi \& F. b. E. N. Parker (Cambridge, UK: Cambridge Univ. Press), 103

Danilovic, S., Hirzberger, J., Riethmüller, T. L., et al. 2014, ApJ, 784, 20

Danilovic, S., Schüssler, M., \& Solanki, S. K. 2010, A\&A, 513, A1

de la Cruz Rodríguez, J., \& Socas-Navarro, H. 2011, A\&A, 527, L8

De Pontieu, B., Carlsson, M., Rouppe van der Voort, L. H. M., et al. 2012, ApJL, 752, L12

De Pontieu, B., McIntosh, S., Hansteen, V. H., et al. 2007, PASJ, 59, S655

de Wijn, A. G., Stenflo, J. O., Solanki, S. K., \& Tsuneta, S. 2009, SSRv, 144,275

Fontenla, J. M., Avrett, E. H., \& Loeser, R. 1993, ApJ, 406, 319

Fontenla, J. M., Curdt, W., Haberreiter, M., Harder, J., \& Tian, H. 2009, ApJ, 707, 482

Gabriel, A. H. 1976, RSPTA, 281, 339

Gafeira, R., Jafarzadeh, S., Solanki, S. K., et al. 2017a, ApJS, 229, 7

Gafeira, R., Lagg, A., Solanki, S. K., et al. 2017b, ApJS, 229, 6

Gandorfer, A., Grauf, B., Barthol, P., et al. 2011, SoPh, 268, 35

Giovanelli, R. G., \& Jones, H. P. 1982, SoPh, 79, 267

Gudiksen, B. V., Carlsson, M., Hansteen, V. H., et al. 2011, A\&A, 531, A154

Hayek, W., Asplund, M., Carlsson, M., et al. 2010, A\&A, 517, A49

Henriques, V. M. J., \& Kiselman, D. 2013, A\&A, 557, A5

Hirzberger, J., Feller, A., Riethmüller, T. L., et al. 2010, ApJL, 723, L154

Hirzberger, J., Feller, A., Riethmüller, T. L., Gandorfer, A., \& Solanki, S. K. 2011, A\&A, 529, A132

Jafarzadeh, S., Solanki, S. K., Feller, A., et al. 2013, A\&A, 549, A116

Jafarzadeh, S., Solanki, S. K., Gafeira, R., et al. 2017b, ApJS, 229, 9

Jones, H. P. 1985, in Chromospheric Diagnostics and Modelling, ed. B. W. Lites (Sunspot, NM: National Solar Observatory), 175

Jones, H. P., \& Giovanelli, R. G. 1982, SoPh, 79, 247

Lagg, A., Lites, B., Harvey, J., Gosain, S., \& Centeno, R. 2015, SSRv https:// doi.org/10.1007/s11214-015-0219-y

Langangen, Ø., De Pontieu, B., Carlsson, M., et al. 2008, ApJL, 679, L167

Leenaarts, J., Carlsson, M., \& Rouppe van der Voort, L. 2015, ApJ, 802, 136

Lemen, J. R., Title, A. M., Akin, D. J., et al. 2012, SoPh, 275, 17

Linsky, J. L., \& Avrett, E. H. 1970, PASP, 82, 169

Low, B. C. 1991, ApJ, 370, 427

Martínez Pillet, V., Del Toro Iniesta, J. C., Álvarez-Herrero, A., et al. 2011, SoPh, 268, 57

Moreno-Insertis, F. 1986, A\&A, 166, 291

Nordlund, A. 1982, A\&A, 107,

Ossendrijver, M. 2003, A\&ARv, 11, 287

Pereira, T. M. D., De Pontieu, B., \& Carlsson, M. 2012, ApJ, 759, 18

Pesnell, W. D., Thompson, B. J., \& Chamberlin, P. C. 2012, SoPh, 275, 3 
Pietarila, A., Hirzberger, J., Zakharov, V., \& Solanki, S. K. 2009, A\&A, 502, 647

Reardon, K. P., \& Cauzzi, G. 2012, American Astronomical Society Meeting Abstracts, 220, 201.11

Rempel, M. 2014, ApJ, 789, 132

Riethmüller, T. L., Solanki, S. K., Barthol, P., et al. 2017, ApJS, 229, 16

Riethmüller, T. L., Solanki, S. K., Hirzberger, J., et al. 2013, ApJL, 776, L13

Roberts, B. 1990, GMS, 58, 113

Rosenthal, C. S., Bogdan, T. J., Carlsson, M., et al. 2002, ApJ, 564, 508

Rouppe van der Voort, L., \& de la Cruz Rodríguez, J. 2013, ApJ, 776, 56

Rouppe van der Voort, L., Leenaarts, J., de Pontieu, B., Carlsson, M., \& Vissers, G. 2009, ApJ, 705, 272

Rutten, R. J. 2006, in ASP Conf. Ser. 354, Solar MHD Theory and Observations: A High Spatial Resolution Perspective, ed. J. Leibacher, R. F. Stein, \& H. Uitenbroek (San Francisco, CA: ASP), 276

Rutten, R. J., de Wijn, A. G., \& Sütterlin, P. 2004, A\&A, 416, 333

Rutten, R. J., Rouppe van der Voort, L. H. M., \& Vissers, G. J. M. 2015, ApJ, 808,133

Schad, T. A., Penn, M. J., \& Lin, H. 2013, ApJ, 768, 111

Schou, J., Scherrer, P. H., Bush, R. I., et al. 2012, SoPh, 275, 229

Schrijver, C. J., \& Title, A. M. 2003, ApJL, 597, L165

Schüssler, M., Caligari, P., Ferriz-Mas, A., \& Moreno-Insertis, F. 1994, A\&A, 281, L69

Sekse, D. H., Rouppe van der Voort, L., \& De Pontieu, B. 2012, ApJ, 752, 108

Sekse, D. H., Rouppe van der Voort, L., De Pontieu, B., \& Scullion, E. 2013, ApJ, 769, 44

Skumanich, A., Smythe, C., \& Frazier, E. N. 1975, ApJ, 200, 747
Solanki, S. K. 1993, SSRv, 63, 1

Solanki, S. K. 2001, in ASP Conf. Ser. Vol. 248, Magnetic Fields Across the Hertzsprung-Russell Diagram, ed. G. Mathys, S. K. Solanki, \& D. T. Wickramasinghe (San Francisco, CA: ASP), 45

Solanki, S. K., Barthol, P., Danilovic, S., et al. 2010, ApJL, 723, L127

Solanki, S. K., Inhester, B., \& Schüssler, M. 2006, RPPh, 69, 563

Solanki, S. K., Riethmüller, T. L., Barthol, P., et al. 2017, ApJS, 229, 2

Solanki, S. K., \& Steiner, O. 1990, A\&A, 234, 519

Solanki, S. K., Steiner, O., \& Uitenbroeck, H. 1991, A\&A, 250, 220

Spruit, H. C. 1981, NASA Special Pub., 450, 385

Stenflo, J. O. 1989, A\&ARv, 1, 3

Stenflo, J. O. 2012, A\&A, 547, A93

Uitenbroek, H. 2001, ApJ, 557, 389

van Noort, M., Rouppe van der Voort, L., \& Löfdahl, M. G. 2005, SoPh, 228, 191

Vögler, A., \& Schüssler, M. 2007, A\&A, 465, L43

Vögler, A., Shelyag, S., Schüssler, M., et al. 2005, A\&A, 429, 335

Wedemeyer-Böhm, S., Lagg, A., \& Nordlund, Å 2009, SSRv, 144, 317

Wiegelmann, T., Neukirch, T., Nickeler, D. H., et al. 2015, ApJ, 815, 10

Wiegelmann, T., Neukirch, T., Nickeler, D. H., et al. 2017, ApJS, 229, 18

Wiegelmann, T., Solanki, S. K., Borrero, J. M., et al. 2010, ApJL, 723, ioppL185

Wiegelmann, T., Solanki, S. K., Borrero, J. M., et al. 2013, SoPh, 283, 253

Wiegelmann, T., Thalmann, J. K., Schrijver, C. J., De Rosa, M. L., \& Metcalf, T. R. 2008, SoPh, 247, 249

Zhang, M., \& Zhang, H. 2000, SoPh, 194, 29 